\title{
O papel necessário da Agência Nacional de Saúde Suplementar na regulação das relações entre operadoras de planos de saúde e prestadores de serviços
}

| ' Silvia Gerschman, ${ }^{2}$ Maria Alicia D. Ugá, ${ }^{3}$ Margareth Portela, ${ }^{4}$ Sheyla Maria Lemos Lima |

Resumo: Este artigo trata do papel regulatório que caberia à Agência Nacional de Saúde Suplementar (ANS) desempenhar, a partir do resultado de um conjunto de pesquisas e estudos sobre as práticas de microrregulação exercidas pelas operadoras de planos de saúde sobre os prestadores hospitalares privados. $\mathrm{O}$ trabalho se baseia em diversos estudos e, especialmente, nos resultados da pesquisa de Ugá et al. (2007), cujos resultados permitiram a problematização de questões relativas à necessidade de regular alguns elementos $\mathrm{da}$ relação entre operadoras e prestadores hospitalares, fundamentais para a garantia do acesso e da qualificação da atenção recebida por clientes de operadoras de planos privados de saúde; e também relativas à importância que os prestadores hospitalares privados possuem para o SUS. Busca-se, assim, a partir dos estudos sobre o tema, refletir tanto sobre o papel regulador a ser desempenhado pela ANS nas relações contratuais entre operadoras e prestadores, quanto sobre o papel que ao SUS caberia exercer.

> Palavras-chave: operadoras de planos de saúde; prestadores hospitalares; Agência Nacional de Saúde; regulação.

\author{
1 Pesquisador titular, \\ Departamento Administração \\ e Planejamento, Escola \\ Nacional de Saúde Pública da \\ Fiocruz. Endereço eletrônico: \\ gerschman@ensp.fiocruz.br \\ 2 Pesquisador titular, \\ Departamento Administração \\ e Planejamento, ENSP/ \\ Fiocruz. Endereço eletrônico: \\ domingue@ensp.fiocruz.br \\ ${ }^{3}$ Pesquisador titular, \\ Departamento Administração \\ e Planejamento, ENSP/Fiocruz. \\ Endereço eletrônico: mportela@ \\ ensp.fiocruz.br \\ ${ }^{4}$ Pesquisador titular, \\ Departamento Administração \\ e Planejamento, ENSP/Fiocruz. \\ Endereço eletrônico: slemos@ \\ ensp.fiocruz.br
}

Recebido em: 19/06/2011. Aprovado em: 31/10/2011. 


\section{Introdução}

Este artigo trata do papel regulatório que caberia à Agência Nacional de Saúde Suplementar (ANS) nas práticas de microrregulação exercidas pelas operadoras de planos de saúde sobre os prestadores hospitalares privados, e que hoje são fundamentalmente direcionadas à redução de custos (pelo constrangimento à utilização de serviços da rede credenciada) e ignoram a questão da qualidade assistencial. Essas práticas, analisadas neste artigo, referem-se às formas de contratualização e, especialmente, à existência ou não de mecanismos sustentados no aperfeiçoamento da qualidade dos serviços oferecidos por esses hospitais à população. Dessa observação, decorrem a análise e a proposta de novos objetos de regulação no segmento da saúde suplementar, apresentados neste trabalho.

Os estudos realizados sobre o tema vêm alimentando uma linha de trabalhos, alguns dos quais já foram publicados e se baseiam na pesquisa "Estudo dos prestadores hospitalares frente às práticas de microrregulação das operadoras de planos de saúde" (UGÁ et al., 2007). Este artigo se baseia nesse e em outros estudos, buscando refletir sobre aspectos relativos à regulação das práticas de assistência à saúde dos prestadores hospitalares privados que prestam serviços às operadoras de planos de saúde. O objetivo é buscar na Teoria da Regulação o modo de entendermos melhor qual o papel, as atribuições e as definições que correspondem à Agência Nacional de Saúde Suplementar (ANS) vis-à-vis à atuação e ao desempenho do respectivo segmento no marco de um sistema de saúde público, universal, baseado no direito de cidadania da sociedade e na obrigação do Estado em providenciá-lo, ou seja, no Sistema Único de Saúde (SUS) tal como definido constitucionalmente.

O trabalho é dividido em quatro partes, relacionadas a seguir: (a) "Considerações conceituais sobre regulação", que se refere à escolha do marco teórico-conceitual a partir do qual é feita a abordagem; (b) "Alguns parâmetros analíticos pertinentes para pensarmos o papel da ANS"; (c) "O papel da Agência Nacional de Saúde e do SUS na regulação do setor privado", que se refere ao marco regulatório adequado à melhoria e ao controle de qualidade dos prestadores hospitalares privados; e (d) "Consideraçôes finais".

\section{Considerações conceituais sobre regulação}

No intuito de introduzir uma discussão específica sobre macro e microrregulação de políticas públicas/sociais enquanto modalidade de intervenção ativa e 
consciente por parte do Estado ou de outras organizações coletivas, cabe tecer algumas considerações fundamentais.

O marco conceitual da regulação, tal como entendido neste trabalho, refere-se ao papel pró-ativo do Estado na definição e intervenção em políticas sociais e, no caso, especificamente, de instrumentos de planejamento de políticas de saúde públicas e privadas. A ideia de regulação aqui utilizada se inscreve na corrente da Escola Francesa de Regulação dos anos 80, que se ocupa das continuidades, das crises e das mudanças históricas das sociedades no capitalismo: como garantir a provisão material e a convivência social sob as condições de produção capitalista. Para isso, é necessário um complexo de instituições e normas sociopolíticas contido de maneira satisfatória pelos conceitos de Mercado e Estado (HIRSCH, 2010).

Importantes referenciais teóricos sustentaram a Teoria da Regulação. Cabe mencionar o papel da Teoria Econômica Institucionalista, da teoria marxista ${ }^{1}$ e da corrente teórica de investigação sócio-histórica, denominada Escola dos Anais (BRAUDEL, 1988).

A Teoria da Regulação estabelece para sua reformulação as conexões necessárias entre a estrutura social objetiva e a ação social consciente. Entende-se por modo de regulação a totalidade de formas institucionais, redes, normas explícitas e implícitas que assegurem a compatibilidade das relações sociais correspondentes às condições materiais de produção e seu desenvolvimento com o consumo social (BOYER, 2004; AGLIETTA, 1976; LIPIETZ apud HIRSCH, 2010).

A noção de regulação propicia a passagem de um conjunto de racionalidades limitadas à possibilidade de coerência dinâmica de um sistema no seu conjunto. $\mathrm{O}$ modo de regulação se refere a todo conjunto de procedimentos que: (a) reproduzam as relações sociais fundamentais; (b) sustentem e conduzam o regime de acumulação; e (c) assegurem a compatibilidade dinâmica de um conjunto de decisões descentralizadas (BOYER, 1990).

A adesão a um conjunto comum de normas morais, jurídicas e econômicas constitui um dos três princípios que definem uma forma institucional; os outros dois são a restrição (seja ela privada ou estatal) e o contrato (individual ou coletivo).

Qualquer forma institucional difunde um princípio de socialização que, ainda que parcial, torna possível a passagem do plano macro ao plano micro. Mas nenhum ator ou grupo desempenha o papel de articulador de sistemas 
diversos, de modo a assegurar, mediante uma ação consciente e deliberada, a estabilização dinâmica da economia.

Todo regime de acumulação/socioeconômico se caracteriza pela forma particular que adquirem os desequilíbrios econômicos e os conflitos sociais (LIPIETZ, 1993). Busca-se, assim, a presença de uma política de regulação econômica social sustentada no papel disciplinador da intervenção estatal sobre interesses econômicos privados, de modo a estabilizar a economia e prevenir conflitos sociais antagônicos. Esta, por sua vez, comporta regulamentações direcionadas a questóes específicas do campo das políticas públicas, de modo que as instituições estatais zelem pela defesa dos interesses públicos sobre os privados.

No campo da Saúde Coletiva, o termo "regulação" é bastante usado, não sempre de maneira adequada. Santos e Merhy (2006) chamam a atenção para a existência de diversas conceituações em regulação, argumentando que a macrorregulação em saúde, que se refere à política pública nesse setor, compreende o próprio processo de formulação e implementação de políticas; as relações entre os setores público e privado; as fontes e mecanismos de financiamento da política; as relações de trabalho no âmbito dos recursos humanos em saúde - ou seja, a contratação, formação e remuneração dos trabalhadores da saúde; as relações com outras políticas sociais -intersetorialidade; e a participação da sociedade controle social. Já a microrregulação é mais direcionada ao dia a dia da assistência à saúde, tanto no setor público quanto no privado.

Ainda que a pesquisa que originou este artigo tenha considerado exclusivamente mecanismos de microrregulação de operadoras de planos privados de saúde em relação à prestação de serviços hospitalares contratados, resultados inesperados nos conduziram a refletir sobre questôes de caráter estrutural, socioeconômicas, e da dinâmica de acumulação do setor econômico de planos e seguros de saúde (ANDREAZZI; KORNIS, 2008), presentes nas relaçōes existentes entre os setores público e privado da saúde no Brasil (SANTOS; UGÁ; PORTO, 1988).

Os resultados dos estudos sobre o setor da saúde suplementar e, dentre estes, a pesquisa referida, foram bastante desafiadores e trouxeram questões da arena macrorregulatória ao evidenciar que é da alçada do setor público, do SUS, por meio da ANS, desempenhar um papel regulador nas relaçôes contratuais entre operadoras e prestadores. Agir nessa direção facilitaria a existência de um conjunto de procedimentos institucionais no SUS capazes de criar, direcionar e 
coibir comportamentos individuais, contribuindo para o ajuste dos mercados, procedimentos esses, por vezes, necessários para o seu próprio funcionamento. Para Polanyi, o mercado não se sustenta sem regras que limitem as consequências do laissez-faire. (POLANYI, 2000).

Entende-se que o modo de regulação sobre a prática contratual e a intervenção de mecanismos adequados para melhorar a qualidade médico-assistencial dos prestadores privados poderiam gerar mecanismos que acionem a totalidade de formas institucionais, redes e normas explícitas e implícitas, capazes de assegurar a compatibilidade das relações no marco de um regime de acumulação de capital - lucro - em correspondência com o estado das relações sociais, mas também transcendendo sua natureza conflitiva (BOYER, 1987).

\section{Alguns parâmetros analíticos}

Os resultados da referida pesquisa e de estudos relativos à regulação do setor de saúde suplementar nos permitiram refletir analiticamente sobre o papel regulatório do Estado, as relações público/privado e a necessidade de intervenção através de agência específica, no caso a ANS, de modo a regulamentar e limitar interesses particulares no campo da Saúde Pública.

A pesquisa (UGÁ et al., 2007) teve por objetivo central conhecer os mecanismos de microrregulação praticados pelas operadoras sobre os prestadores de serviços hospitalares, bem como a forma como eles repercutem nas práticas dos hospitais. Ela traz também informações referentes ao parque hospitalar privado que presta serviços às operadores de planos de saúde no Brasil. Para isso, foi desenvolvido um trabalho de campo, efetuado por pesquisadores treinados, em uma amostra constituída de 74 hospitais disseminados no território nacional, a partir de um universo de 3817, e.estratificada por macrorregiōes geográficas e por tipo de hospital.

Os principais resultados dessa pesquisa apontam que:

- o parque hospitalar privado que presta serviços às operadoras de planos de saúde é composto, na sua maioria, por unidades de pequeno porte; dessas, $72 \%$ integram a rede de prestadores do SUS;

- há uma forte concentração, tendência ao monopólio no mercado de operadoras de plano de saúde; 
- predomina, nesses hospitais, uma baixa presença de estruturas e práticas de qualificação da gestão assistencial, sendo que, nos que prestam assistência ao SUS, essa presença é menor ainda;

- os hospitais privados que integram o SUS tendem a ser os que menos investem na qualidade assistencial;

- nesses hospitais, o monitoramento de indicadores centra-se preponderantemente na questão da produção - tempo médio de permanência e taxa de ocupação de leitos;

- os indicadores de resultados da assistência acompanhados mais frequentemente são somente a taxa de infecção hospitalar e a taxa de mortalidade geral;

- os indicadores mais precisos de qualidade da gestão assistencial mostram baixíssima ocorrência de práticas mais apuradas de gestão da clínica;

- é baixíssima, quase nula, a microrregulação que as operadoras de planos de saúde exercem sobre os hospitais em termos da qualificação da assistência;

- trata-se de um enorme conjunto de prestadores hospitalares privados que não se percebem como pertencentes a nenhuma rede de prestadores privados;

- os contratos efetuados entre operadoras e prestadores são meramente comerciais;

- não há preocupação explícita com as instalações dos hospitais nem com a qualidade da atenção oferecida;

- dá-se pouca importância aos aspectos legais obrigatórios a serem preenchidos pelos hospitais e relativos ao Cadastro Nacional de Estabelecimentos de Saúde, à Vigilância Sanitária e ao Sistema de Qualificação da Agência Nacional de Saúde Suplementar.

Esses achados coincidem com os de outra pesquisa, publicada pela ANS (ANS, 2005), na qual é estudado o mesmo fenômeno, o da microrregulação das operadoras de planos de saúde sobre os prestadores de serviços, a partir de uma amostra de conveniência, abrangendo importantes empresas que atuam no segmento da saúde suplementar. Seus principais resultados apontam que:

- no que tange às relaçóes administrativas, financeiras e comerciais entre operadoras e prestadores, os pontos mais críticos são os fluxos de pagamento (inadimplência, glosas), a utilização de órteses e próteses, e a incorporação de tecnologias de alto custo, dentre outros;

- no que concerne à transição tecnológica e à prática médica, verificou-se um processo que consubstancia uma verdadeira reestruturação produtiva da prática 
médica, pois esta se confronta com a microrregulação introduzida sobre os mé-

dicos pelas práticas da atenção gerenciada. Nesse sentido, segundo os autores, pode-se estar vivendo uma transição tecnológica no setor saúde, caracterizada não mais pela incorporação de tecnologias duras ao processo de cuidado, mas por uma verdadeira tentativa de captura de autonomia dos médicos, através de sofisticados mecanismos gerenciais direcionados à contenção de custos.

Ainda, considerando outros estudos, verifica-se que a regulação desse setor vem adquirindo uma enorme complexidade, já que as empresas criadas pelo capital financeiro utilizam numerosos artifícios jurídicos para encobrir sua atuação, fracionando-se em numerosas companhias sem conexão (IRIART, 2008). Ainda, segundo Gerschman (2008), a não existência de políticas de microrregulação das operadoras, nem do SUS, favorece a segmentação e especialização do mercado de serviços hospitalares privados sem que haja de uma saudável competição no aperfeiçoamento, na qualificação e qualidade dos serviços ofertados pelo segmento hospitalar privado.

Considerando os elementos acima apontados, cabe perguntar sobre a forma como a ANS poderia intervir na relação contratual mantida entre operadoras e prestadores de planos de saúde sem que esta se torne uma atribuição indevida ou não legítima entre relações institucionais da esfera privada. Quais as funções e atribuiçôes regulatórias que caberiam ao sistema público de saúde sobre o parque hospitalar privado de serviços de saúde, e, especificamente, sob que condição o SUS poderia exercitá-las sem configurar interferência indevida?

Ao se constatar a inexistência de mecanismos regulatórios utilizados pelas operadoras de planos de saúde na contratação de prestadores de serviços hospitalares, inclusive os mecanismos relativos às modalidades em que se exercem as práticas desses serviços e, fundamentalmente, ao fato de se tratar de hospitais na sua maioria prestadores de serviços ao SUS, fica evidente a dimensão em que esses serviços podem ferir o direito à atenção integrada e à qualidade da saúde pública.

\section{O papel da Agência Nacional de Saúde na regulação do setor privado}

Após as considerações levantadas, cabe refletir sobre a expansão do papel da ANS em relação à ausência de regulação que as operadoras exercem sobre os 
prestadores e sobre a necessidade de monitoramento da atuação de ambos no que se refere à atenção à saúde dos cidadãos

De modo geral, as funções básicas das agências regulatórias, especificamente da ANS, podem ser sintetizadas em:

- garantir que os contratos entre operadoras e beneficiários sejam cumpridos, ou seja, que não exista nenhum tipo de interferência política ou de qualquer outra ordem que afete ou inviabilize o seu cumprimento;

- garantir o fornecimento regular com baixa interferência no serviço prestado;

- tornar-se parceira da qualidade do serviço prestado.

Além dessas, a ANS tem uma característica específica que a diferencia de outras agências regulatórias, que é a de lidar com um bem de mérito, que em nenhum caso poderia tornar-se mercadoria. Sua esfera de atuação é a proteção, o cuidado e a conservação da vida humana, a que todos têm direito..

A pesquisa mostrou que, do ponto de vista mercantil, as operadoras funcionam como é de se esperar, maximizando renda. Contudo, por serem os serviços hospitalares bastante diferenciados de outros serviços públicos, as operadoras deveriam exigir do prestador boa qualidade do serviço contratado. Mas os resultados da pesquisa são claros a respeito da ausência de regulação quanto à qualidade da assistência exigida pela operadora na sua relação com os prestadores hospitalares.

Considerando que as operadoras necessariamente têm que oferecer baixos riscos na atenção à saúde, seria possível demandar delas garantias de que o prestador atue dessa forma. Essa exigência contribuiria, alem do mais, para evidenciar a seriedade comercial da operadora perante a ANS.

O critério básico desta para operar sobre a dispersão enorme de pequenas operadoras sem nenhuma condição econômico-financeira para funcionar não pode conduzir à noção de que há duas modalidades de regulação: uma que se refere aos aspectos contratuais entre operadoras e prestadoras e outra que diz respeito à qualidade da assistência. Se a prioridade da operadora é o contrato comercial com o prestador, ela deveria determinar o tipo de serviço a ser prestado. Ou seja, como no momento a relação mercantil funciona bem para as operadoras, ela acaba por tornar-se o critério dominante na sua relação com os prestadores, sendo descartada a regulação relacionada à atenção à saúde dos usuários. É levado em conta apenas o aspecto comercial da saúde. 
Outro assunto de importância se refere à enorme concentração existente entre operadoras e prestadores hospitalares privados. Praticamente $80 \%$ dos hospitais têm contratos com a Unimed. Ou seja, a maioria dos hospitais presta serviços para uma mesma operadora. Isso significa que, na medida em que a prestação é concentrada em um número pequeno de operadoras de planos de saúde, ela se acomodará às exigências requeridas por elas. Assim, se não for exigido do hospital, no momento da contratação, melhor qualidade de infraestrutura, de serviços, de práticas profissionais, de atenção e de gestão, os prestadores hospitalares não serão obrigados a obedecer padrões mais apurados de atenção. Sem a obrigatoriedade de melhor atendimento à saúde dos beneficiários, passam a ser as principais operadoras (a Unimed, a Cassi e a Geap), que dominam o mercado de prestadores, as que definem a qualidade da assistência no pais. De fato, a presença monopólica dessas três operadoras evidência a enorme concentração de mercado.

Da enorme dispersão anterior, quando proliferavam pequenos e insolventes planos e seguros, passou-se a uma redução drástica devido à atuação regulatória da ANS, sendo infelizmente substituída - consequência não antecipada da regulação - por grande concentração.

Três operadoras passam a exercer sobre os prestadores hospitalares uma influência muito grande. Na realidade, o monopólio é quem regula, de fato, o papel dos hospitais nessa forma de interação entre as partes. Observe-se, de uma parte, o avesso da pulverização dos planos de saúde, que existia antes da regulação da ANS e que propiciava a prática irregular, quando não delituosa de securitização (que pode ser ilustrada por operadoras que se constituíam apenas de uma ambulância para levar o paciente ao hospital do SUS). Mas, de outra parte, verifica-se agora que poucas operadoras concentram a contratação da maior parte dos prestadores hospitalares, o que poderia conduzir, por conta do enorme poder mercantil que exercem, à regulação transversal em saúde. Uma grande operadora, não obstante a segurança que oferece, pode pôr-se no lugar da agência regulatória.

A ANS já vem conseguindo grandes avanços das operadoras enquanto entidades mercantis. Agora, é preciso impedir que esse poder comercial se transforme em força regulatória sobre os prestadores e usuários. Para qualificar ou requalificar a forma da lei que regula as operadoras, é necessário introduzir critérios substantivos não apenas quanto à lisura das operações comerciais, mas 
também quanto à prestação dos serviços de saúde. Esses critérios deveriam ser parte dos processos de acreditamento das operadoras pela ANS.

Entre os aspectos definidos pelos estudos destinados a aferir a qualidade assistencial dos provedores hospitalares que prestam serviços às operadoras de planos de saúde, selecionamos aqueles que não poderiam faltar às práticas hospitalares. Nesse sentido, a proposta é de uma regulação progressiva, que deveria iniciar-se por aqueles aspectos e indicadores que não poderiam estar ausentes da atenção hospitalar até completar a totalidade dos aspectos e indicadores analisados pelos estudos que serviram de referência ao presente trabalho, e atingir a qualidade assistencial desejável para o setor hospitalar privado (PORTELA et al, 2008; 2010). Cinco seriam os indicadores escolhidos: a qualificação da gestão; a presença de estruturas e práticas de garantia e monitoramento da qualidade da assistência; as práticas de gestão da clínica; a presença de sistema de informação; e a exigência de acompanhamento de indicadores.

Ainda no que se refere aos prestadores hospitalares que são financeiramente alimentados por essas grandes operadoras e pelo SUS, fica evidente serem os piores classificados pela pesquisa. Isso denuncia o papel omisso do Estado em relação aos hospitais que financia. Para situações desse tipo, a ANS deveria ter instâncias de regulação imediata e conjunta com o Ministério da Saúde/SUS, pois, ao não regular aspectos substantivos dos serviços, indiretamente se conferem incentivos às operadoras privadas para fortalecer prestadores hospitalares que demonstram baixa classificação, prejudicando o SUS. Da mesma maneira, a ANS poderia também atuar conjuntamente com a Agência Nacional de Vigilância Sanitária (ANVISA) no que se refere a indicadores de infecção hospitalar e outros, assim como em questôes relativas a medicamentos, alimentação, higiene, preservação de instalações e outros.

Sob essa perspectiva, as operadoras seriam induzidas a estabelecer um contrato a partir do qual o hospital preste um serviço de qualidade ao usuário, e não apenas um conjunto de clausulas comerciais. Aliás, uma questáo que aparece como demanda dos prestadores é a solicitação de um contrato padrão por parte das operadoras. Trata-se de um equívoco, dadas as diferenças existentes entre os prestadores. A regulação deveria considerar, no contrato, sob a forma de atuação normativa, a especificidade dos serviços de saúde e a qualidade básica a ser prestada. Dessa forma, as operadoras que são beneficiárias do acreditamento na 
ANS e que como tal se apresentam seriam corresponsabilizadas pela qualidade dos serviços do prestadores contratados.

Os planos privados de saúde não são um seguro qualquer, e por esse motivo devem envolver cláusulas específicas.

\section{Considerações finais}

A ideia de interpretar os mecanismos e relações existentes entre as operadoras de planos privados de saúde e o setor hospitalar privado prestador de serviços sob a perspectiva da Teoria da Regulação sustenta-se nos resultados de estudos que evidenciam que a maior parte do setor não apenas presta serviços médicoassistenciais aos beneficiários de planos de saúde, mas também o faz aos usuários dos sistema público. Esse fato exige pensar nexos regulatórios capazes de operar em contextos complexos.

A incorporação da Teoria da Regulação teve por função considerar um certo tipo de comportamento econômico dos grupos ou indivíduos em questão. Tratase de um processo de transmutação dos direitos individuais em direitos coletivos, que está bastante presente em vários setores do direito trabalhista, comercial e administrativo. Mas, na realidade, a adesão a um conjunto comum de normas jurídicas e econômicas constitui apenas um dos princípios definidores de uma forma institucional. Os outros dois são representados pela restrição privada ou estatal e pelo contrato individual e coletivo.

Por se tratar de planos privados de saúde, cumpre não separar a operação mercantil da prestação - afinal trata-se de um setor privado da economia. Contudo, não se deveria admitir a prevalência, muito menos a exclusividade, dessa dimensão. Se isso ocorre, as grandes operadoras arrogam-se a ocupação do lugar regulatório que pertence, de direito, à ANS. É atribuição desta agir de maneira a criar normas de acreditamento que obedeçam tanto à saúde mercantil quanto à própria natureza do contrato entre operadoras e prestadores. Devem-se respeitar as exigências de natureza específica, assim como as relativas à qualidade dos serviços prestados.

Acredita-se que a formação de uma comissão nacional que envolva o Ministério da Saúde, a Agência Nacional de Saúde e a Agência Nacional de Vigilância Sanitária seria de primordial importância para regular o setor hospitalar privado conveniado com o SUS e contratado pelas operadoras de planos de saúde. 
Essa comissão teria a incumbência de acompanhar questões e indicadores da qualidade da assistência, e ampliar o escopo de intervenção e regulação, tendo como norte abranger completamente o diagnóstico resultante da pesquisa. ${ }^{2}$

\section{Agradecimentos}

As autoras agradecem à Agência Nacional de Saúde Suplementar (ANS) e à Organização Pan-Americana da Saúde (OPAS/OMS) o apoio para a realização do estudo no qual este artigo se baseia. As autoras agradecem, ainda, in memoriam, ao Prof. Miguel Murat Vasconcellos, que fez parte da equipe do projeto e participou da concepção da pesquisa que originou este artigo. Ele faleceu antes de sua conclusão, enorme perda que muito lastimamos.

\section{Referências}

AGÊNCIA NACIONAL DE SAÚDE SUPLEMENTAR. Duas faces da mesma moeda: microrregulação e modelos assistenciais na saúde suplementar. Rio de Janeiro ANS, 2005.

ANDREAZZI, M.F.; KORNIS, G. Padrōes de acumulação setorial: finanças e serviços nas transformações contemporâneas da saúde. Ciencia e Saúde Coletiva, v.13, n.5, p.14091421, 2008.

ANGLIETTA, M. Régulation et crises du capitalisme. Paris: Calmann-Lévy, 1976.

BOYER, R.; MISTRAL, J. Accumulation inflation, crises. Paris: PUF, 1987.

BOYER, R. A Teoria da Regulação: uma análise critica. São Paulo: Nobel, 1990.

BOYER, R. Théorie de la régulation, 1. Les fondamentaux. Paris: La découverte, 2004 (Collection Repères).

BRAUDEL, F. La dynamique du capitalisme. Paris: Flammarion, 1988.

GERSCHMAN, S. Políticas comparadas de saúde suplementar no contexto de sistemas públicos de saúde: União Europeia e Brasil. Ciência e Saúde Coletiva, v.13, n.5, p.14411452, 2008.

HIRSCH, J.Teoria Materialista do Estado. Processo de Transformação do Sistema capitalista de Estados. Rio de Janeiro: Revan, 2010.

IRIART, C. Capital financiero versus complejo médico-industrial: los desafios de lãs agências regulatórias. Ciencia e Saúde Coletiva, v.13, n.5, p.1619-1626, 2008.

LIPIETZ, A. From Althusserian to Regulation Theory. In: KAPLAN, A.; SPRINKER, M. (Eds.). The Althusserian Legacy. London: Verso, 1993. 
POLANYI, K. A grande transformação. As origens da nossa época. 6 ed. São Paulo: Campos, 2000.

PORTELA, M.C. et al. Diretrizes clínicas e outras práticas voltadas para a melhoria da qualidade assistencial em operadoras de planos de saúde sob a perspectiva dos seus dirigentes no Brasil. Caderno de Saúde Pública. Rio de Janeiro, v.24, n.2, 2008.

PORTELA, M.C. et al. Estrutura e qualidade assistencial dos prestadores de serviços hospitalares, Ciência \& Saúde Coletiva. Rio de Janeiro, v.26, n.2, 2010.

SANTOS, I.; UGÁ, M.A.D.; PORTO, S. O mix público-privado no sistema de saúde brasileiro: financiamento, oferta e utilização de serviços de saúde. Ciência \& Saúde Coletiva. Rio de Janeiro, v.13, n.5, p. 1431-1440, 1988.

SANTOS, F.P.; MEHRY, E.E, A regulação pública de saúde no Estado Brasileiro- uma revisão, Interface, n. 18, 2006.

UGÁ, M.A.D. et al. Estudo dos prestadores hospitalares frente às práticas de microrregulação das operadoras de planos de saúde. Relatório de Pesquisa. Rio de Janeiro, 2007.

\section{Notas}

${ }^{1}$ Esta teve na França uma importante influência da teoria estruturalista de Althusser (LIPIETZ, 1993).

${ }^{2}$ S. Gerschman elaborou o desenho do estudo, das análises e da redação do manuscrito. M.C.Portela e S.M.L.Lima participaram no desenho do estudo, nas análises e na revisão do manuscrito. M.A.D.Ugá colaborou na coordenação da pesquisa, no desenho do estudo e na elaboração e revisão do manuscrito. 
The necessary role of the National Supplementary Health Agency in the regulation of relations between managed care companies and service providers

This paper discusses the regulatory role that would fit the National Supplementary Health Agency (ANS), from the results of researches and studies on the practices of micro-regulation performed by private managed care companies on hospital providers they contract. It is based on several studies, especially on the research by Ugá el al (2007), whose results allowed the questioning of issues related to the necessary regulation of some elements of the relationship between health managed care companies and hospital providers, key to ensuring access and qualification of health care received by customers of those companies, as well as the importance that hospital providers have for the public health system, SUS. The paper aims, therefore, from the studies on the subject, to reflect on the regulatory role to be played by ANS in the contractual relations between managed care companies and providers and on the role that would fit to the SUS on this matter.

> Key words: health regulation; managed care companies and private hospitals; regulation theory. 


\section{ERRATA}

Caros leitores de Physis,

Por motivos alheios a nossa vontade, o último número (v. 22, n. 2) de nossa revista foi impresso com dois pequenos erros: primeiramente, o título correto do tema que deveria constar na capa é Políticas de Saúde e Gestão. Em segundo lugar, o nome completo da quarta autora do artigo "O papel necessário da Agência Nacional de Saúde Suplementar na regulação das relações entre operadoras de planos de saúde e prestadores de serviços" (p. 463-476), é SHEYLA MARIA LEMOS LIMA. 